\title{
ВMJ Global Health Reshaping public hospitals: an agenda for reform in Asia and the Pacific
}

To cite: Gauld R,

Asgari-Jirhandeh $\mathrm{N}$,

Patcharanarumol W, et al.

Reshaping public hospitals: an agenda for reform in Asia and the Pacific. BMJ Glob Health 2018:0:e001168. doi:10.1136/ bmjgh-2018-001168

Handling editor Seye Abimbola

Received 14 September 2018 Revised 15 October 2018 Accepted 23 October 2018

\section{Check for updates}

\section{(C) Author(s) (or their} employer(s)) 2018. Re-use permitted under CC BY-NC. No commercial re-use. See rights and permissions. Published by BMJ.

${ }^{1}$ Otago Business School, University of Otago, Dunedin, New Zealand

${ }^{2}$ Asia-Pacific Observatory on Health Systems and Policies, World Health Organization, Delhi, India

${ }^{3}$ International Health Policy Program, Ministry of Public Health, Bangkok, Thailand

Correspondence to Professor Robin Gauld; robin.gauld@otago.ac.nz

\section{ABSTRACT}

Hospitals in the Asia-Pacific today face the 'triple aim challenge, proposed by the Institute for Healthcare Improvement, of how to improve quality of care and population health, while at the same time controlling healthcare costs. Yet, pursuing these challenges in combination is presently a remote prospect for many hospitals and, indeed, in a majority of countries in the region. The roles and functions of the public hospital sector within local health systems need redefinition and reform in the context of demographic and epidemiological transitions. Policymakers, managers and health professionals have an obligation to reshape the future of public hospitals. This article outlines actions for how public hospitals can be reshaped from a health system perspective. First, hospitals should be integrated into the fabric of the local health system; they can lead in this through working in alliances with other healthcare facilities, including primary care and private hospitals. Policymakers have a role in facilitating this as it contributes to health improvement of the population. Second, investments in system innovation, management improvement and information systems are required and their impact assessed. Such investments can contribute to cost control and efficiency. Public hospital sector investments should be strategic, efficient and should not bias investment in broader determinants of health. Third, reorienting health workforce competencies and appropriate skills should be central to hospital sector reforms, from policy to frontline services delivery. Creative thinking is needed to build and support flexible care delivery arrangements for services designed to respond to patients ' and providers' needs. Pivotal to achievement of each of these three areas of reform is good governance and leadership.

\section{INTRODUCTION}

What should be the future role of the public hospital sector in the health systems of Asia and the Pacific and how might policymakers and health professionals work to shape this? As with other parts of the globe, the region's hospitals-public as well as private-face the 'triple aim' challenge of how to improve quality of care, improve population health and contain costs. ${ }^{1}$ For many hospitals, specifically in the public sector, the pursuit of these
Summary box

In many Asian and Pacific jurisdictions, the public hospital sector is underdeveloped and not able to meet changing community needs due to insufficient financial and policy support, poor or perverse incentives and workforce challenges.

- Forging an agenda for public hospital sector reform is a critical development issue, particularly given rapidly changing demographics towards societal ageing and increasing non-communicable disease with multiple morbidities.

- The reform agenda should include use of the 'triple aim' of improved quality of care, population health and cost containment to guide planning, along with strategic investments in three areas: in alliance governance; leadership around innovation and improvement and in workforce redesign with interprofessional education and practices key to this.

- In the absence of reform, there is considerable risk that public hospitals and broader health systems within which they are located will fail to meet future healthcare needs of the population.

three aims remains a remote prospect. This is because: hospital funding has developed along a trajectory that provides periodic treatment of individual patients with limited links with broader health systems and inadequate responses to community and population health needs; funding incentives are often oriented towards inpatient treatment and concepts of competition rather than collaboration have tended to be central to activities of public hospitals, driving behaviour that is counter to the whole system approaches that future healthcare needs will demand. ${ }^{2}$

With the rapidly changing population and disease profiles of Asia and the Pacific, policymakers, managers and health professionals have an obligation to reshape public hospitals and their role in the health system. ${ }^{3}$ They need to take a systems perspective in which hospitals are seen as a component of the broader health system-a critical component-but not independent from or dominating the system. ${ }^{4}$ Viewed this way, public 
hospitals, often the key providers of advanced medical care and technology and comprehensive care, should be the location for specific kinds of patients who are not able to be treated elsewhere. As part of the system, public hospitals should work closely with other providers to ensure treatment in the right place, at the right time and by the right provider. Policymakers need to develop and promote effective systems for referral between hospital and primary healthcare to improve patient outcomes and efficiency.

This article outlines an agenda for reshaping the public hospital sector, based on three key components: hospitals should be integrated into the fabric of local health systems through an alliance governance model with strong community linkages; they should be leaders on, and strategic investors in, innovation and they have an opportunity to develop and implement new health workforce configurations and influence debates around training and regulation of health professionals. The article details this agenda, including why the three components are critical, why public hospitals are best placed to provide leadership on them and how committing to implementing the three components can contribute to achieving the triple aim.

\section{WHY THE TRIPLE AIM?}

Introduced in 2008 by the Institute for Healthcare Improvement, the triple aim brings together the fundamental issues that many health system leaders around the globe are concerned about. The essential argument is that, if all three aims are kept in focus, then overall system performance will naturally improve. ${ }^{15}$ If health policy and systems focus on a population rather than simply the individuals who seek care from within a population, then this will lead to population-based planning-the first of the three aims. The focus will tilt towards identifying health determinants that contribute to disease in the population and initiatives for alleviating these. This will, in turn, shift care providers increasingly to work with others in the community able to influence these factors, many of which may sit outside of the direct influence of healthcare. ${ }^{4}$ An example is effective interventions for tobacco control such as raising tax, increasing the retail price and restricting advertising. The second aim is that providers-especially of hospital care-make quality improvement a priority to ensure patient safety and favourable clinical outcomes, with recognised approaches to quality improvement applied to care design and delivery. Aim number three, cost containment, is an outcome of the first two aims. A growing body of literature demonstrates the successes that can result from pursuit of the triple aim. ${ }^{5-7}$ Notably, the framework was developed in the US context of private healthcare delivery where the idea of focusing on a population and community care is almost anathema to inpatient care and revenue growth. Yet, it has had impact in that country and in others with predominantly public hospitals and public funding.
The framework is, arguably, applicable to hospitals and health systems in Asia and the Pacific; indeed, it has been deployed in Singapore and New Zealand as well as parts of Australia and Japan. ${ }^{7}$

\section{THE REFORM AGENDA}

Hospitals in Asia and the Pacific along with policymakers and others have an opportunity, even obligation, to invest strategically in reforms for a future set of directions that look different from the present developmental path in many of the region's countries. This present pattern is one of public hospitals often a step behind their private counterparts in terms of agenda setting, patient perceptions and quality of care; of hospitals operating in isolation from one another and the rest of the health system, including primary care, with development ad hoc and passive responses to growth in healthcare demand and of governance mostly oriented towards hospital-specific and, especially, financial performance. ${ }^{289}$ Reform attempts, particularly those aimed at improving primary care, which are known to be associated with improved health system performance, ${ }^{10}{ }^{11}$ are often frustrated by hospital and medical specialist resistance and their dominant role. ${ }^{12}$ Public hospitals can lead in reversing this. The next sections elaborate on the fundamentals of a reform and investment strategy that includes developing alliance governance, investing in innovation and reorienting the health workforce.

\section{Alliance governance for integration}

There is an emerging global consensus that system integration is critical for meeting the challenges of ageing patients, non-communicable diseases and multimorbidity-the patients who, into the future, will potentially overwhelm the health systems of Asia and the Pacific. ${ }^{13-15}$ Integration here means that the providers of different services are organised according to the needs of such patients and work together in a system - a patient-centred approach. ${ }^{16-19}$ Most of the aforementioned patients require more than the traditional periodic clinical encounters with an individual doctor or nurse-working in a hospital or primary care-focused on a specific problem; their care requires a different method for governing, designing and organising services which ensures that different providers work collaboratively for the whole system not just their own service..$^{20}$ Their care requires stronger primary care, with hospitals supporting and facilitating care outside their walls. This includes shifting the locations of service delivery from hospitals to community settings where appropriate as well as shifting responsibility for care provision away from hospital specialists to appropriately qualified and supported generalists and primary care providers.

Alliance governance offers the best method for facilitating the discussions required for such shifts in the provision landscape. ${ }^{21}$ Alliance governance has been implemented throughout New Zealand, parts of Australia 
and elsewhere around the world in direct response to the health system challenges' future patients present. An effective alliance consists of leaders from across the care spectrum, including both health professionals and managers. It therefore brings together the managers from different services along with clinical leaders, emphasising the latter as robust clinical leadership is necessary for care redesign and system integration. An alliance governance group may feature, according to health system context, doctors, nurses and allied professionals from primary care and hospitals along with others in the health system such as ambulance and aged care residential services. The private sector should also participate. Patient representatives, of course, should also be involved for the reason that health systems of the future should be designed around the needs of patients rather than providers of services. With such membership, it is possible to have conversations around who, in the local system, should be responsible for which components of patient care and what resources are required from resulting collaborations and agreements for service shifts. ${ }^{22}{ }^{23}$ For example, if primary care doctors are to take a lead role in managing multimorbidity and patients non-communicable disease, then what additional training and support from hospital colleagues, along with resources previously provided for such patients in the hospital sector, might be needed? Which other primary care providers will be able to assist with this? And what does the perspective and preferences of patients and their experiences with the system, which is often revealing, bring to the care design process?

In terms of structure, an alliance governance group may delegate activities to a series of clinical networks, each charged with responsibility for integration and care redesign in their area of responsibility. ${ }^{21}$ Networks may be established in areas such as older persons' health, mental health, long-term conditions, outpatients or where an alliance determines creating a clinical network could be beneficial. Composition should be similar to that of an alliance governance group, including professionals, managers and patient representatives. Ideally, a clinical network should be given a mandate for members to work together to change the system on behalf of patients. Research shows that effective alliance governance requires alternative or innovative funding models, which provide incentives for the different partners to share resources and support one another and achieve health improvement and efficiency. ${ }^{24} 25$ Underpinning this is the need to build strong relationships and trust between partners.

As key players, often at the centre of healthcare delivery in a locality, public hospitals in the respective countries of Asia and the Pacific have potential to lead alliance development. They have an opportunity to transform governance arrangements, with members entering into an alliance on the understanding that they are there to share resources and work collaboratively with one another to build integrated systems on behalf of their communities. Alliances have been shown to be effective, improving efficiency as well as patient experiences and clinical leadership. They have also produced service delivery innovations that can only come when different providers in the spectrum of care agree to work collaboratively, putting the patient at the centre of discussions. ${ }^{26} 27$

\section{Leaders and investors in innovation}

Globally, there has been considerable attention in recent years to innovation in health systems. ${ }^{28-30}$ By innovation, this means that care design and delivery, and facilitators of this, require remodelling through application of new approaches and methods for delivery. Yet, this cannot happen without leadership, commitment to change and investment. ${ }^{31}$ Very importantly, and following the triple aim, investments in public hospital innovation should be strategic and not at the expense of population health investments. Indeed, innovation investment may either need to be a budgetary supplement or redirected from within existing budgets on the understanding that resulting innovations will produce long-term financial benefits and quality improvements. This, of course, is in keeping with the triple aim as well as the findings of various innovation and improvement studies. ${ }^{532}$

For many public hospitals and funders in the Asia-Pacific region, especially where insurance is the dominant funding source, innovations have been on funding methods to achieve universal health coverage and strategic purchasing to achieve improved efficiency and cost containment. To achieve universal health coverage, this again highlights the importance of primary care and improved support from public hospitals. The next phase of reform and investment needs to be in system improvement. This should focus on three system levels.

First, innovation and improvement methods need to be applied to all services. ${ }^{28}{ }^{33}$ Underpinning this is the intent to reduce variation in clinical practice, patient experiences and outcomes. Suggestions are that around $75 \%$ of hospital care delivery is amenable to standardisation, with the remainder being more complex cases. ${ }^{34}$ For relatively standard cases of conditions such as pneumonia and stroke, standard clinically agreed procedures can be implemented. ${ }^{32}$ Patients and providers can know exactly what to expect from the system and what needs to be done at every step in a patient's hospital journey. The key is to have mechanisms in place to identify non-standard cases early and concentrate on reducing wasted time and resources in the care process and agreeing to and documenting all clinical processes to be delivered in standard cases, based on the best available evidence, with an expectation that all health professionals will adhere to treatment guidelines. Information systems can assist standardisation and compliance with processes, including providing alerts when care falls outside of agreed protocols. ${ }^{35}$ When linked with referrals between primary care and hospital services, such systems and associated care redesign processes have been shown, in Thailand, to improve management of patients with chronic disease. ${ }^{36}$ 
Second, and pivotal to delivering on the previous point, investments should be made in clinical leadership training. Studies have shown that strong clinical leadership contributes to better care quality and financial performance and a more positive workforce. ${ }^{37-39}$ Yet, most health professionals receive limited leadership input in their clinical training, and leadership continues to be viewed as 'step down' from clinical duties. ${ }^{40} 41$ Hospitals should commit to leadership training across the entire workforce, combining generic managers and health professionals in this. The future is also one where clinical leadership should be seen as conterminous with high-quality care delivery. In this regard, professionals should be taught that they have two jobs and supported in this: to be highly skilled, safe and competent practitioners and to be constantly focused on improving the system in which they work. For the latter, training is critical and needs to incorporate improvement science, organisational leadership, human resource management and other administrative disciplines. ${ }^{40}$

Third, substantial investment is needed in information systems. The professionals and patients of tomorrow will be reliant on and expect to maximise benefit from the use of personal mobile technology for healthcare services. The potential for engaging patients in self-care and with the system is great as is the potential for traversing the barriers between different parts of the health system. Few systems around the world have captured this potential. The relatively underdeveloped systems in many parts of Asia and the Pacific offer scope for investing in the next generation of information and technology systems. This means thinking beyond the static desktop systems of today towards bringing together personal health, system and professional information. Industry has traditionally driven information system development, at high cost to public hospitals and with high project failure rates. ${ }^{42}$ There is an opportunity for clinical and hospital leaders to drive their own information system projects, investing in their own staff in the process. There is scope for the public hospitals of the region to develop systems that could be, in turn, commercialised.

There are numerous examples globally where hospitals and others in the health system have invested in mechanisms specifically intended to support innovation. The English National Health Service invested in 13 'CLAHRCs': Collaborations for Leadership in Applied Health Research and Care, which are academic and hospital innovation partnerships, with an aim of bringing rigorous research to the innovation process with a focus on key questions of importance to health improvement. ${ }^{43}$ Similarly, US hospitals have invested in independent innovation initiatives with an express goal of driving innovation efforts, measuring progress and disseminating results. ${ }^{32} 33$

\section{Designers of the future workforce}

One of the most pressing issues for health systems today is the need for a professional workforce configured to respond to future healthcare needs. ${ }^{44}$ In particular, to be able to deliver care in the context of increasing multimorbidity and population ageing. As with the rest of the globe, the countries of Asia and the Pacific continue to train professionals and organise service delivery as they have done for decades. This involves training students in medicine, nursing, pharmacy and other allied professions largely in separation from one another with the focus predominantly on competence in the professional area. This has been mirrored in practice, with organisation of hospital and non-hospital services and the culture of different professional groups often making teamwork and collaboration difficult, in turn, exacerbating the fragmentation of care delivery.

There is routinely a hierarchy in hospitals and health systems, with medical specialists at the apex. Asia's health systems are far from immune to this, with students continuing to aspire to specialisation and their teachers continuing to project an aura of expert authority and social status which should never be questioned. While not to undermine the importance of specialised health professionals, the future requires a different model and approach to workforce training and design and a new professional ethos. ${ }^{445}$ In this, public hospitals, often the sites for training, can provide leadership.

Two broad options need to be pursued. First, hospitals can lead on discussions around organisation of care. Ideally, care should be provided not by individual specialists, with nurses and other professionals in support, but by interprofessional teams. ${ }^{47}$ This requires a shift in thinking among professional groups around how they work together and support one another. Hospitals can lead in providing training for team work which tends not to be central to professional training, particularly in medicine. Such training should extend beyond the hospital to include community providers. ${ }^{48}{ }^{49}$ In this way, the focus will gradually shift to the role that different professionals play in the care process and improvements needed when the team fails a patient. Often when a patient is harmed or subject to substandard care, this is due to a lack of team care or professional collaboration. ${ }^{50}{ }^{51}$ Team care should be focused on ensuring every professional understands their place in the system of care, including upholding agreed care processes and standards, with team members actively scrutinising and reflecting on one another's work. The role of medical specialists and other professionals in the system ought to be on supporting colleagues in primary care settings and vice versa, with each practitioner in the system consistently asking how they can do better in this regard. For hospital doctors, this includes training in primary care to improve understanding of this setting. Pharmacists can also play a crucial role in working with professional colleagues and patients to improve understanding of medicines. Nurse practitioners, with prescribing rights, can work with patients who, traditionally, have required a medical consultation. Hospitals can lead in developing a 'pact' between hospital leaders and professionals to 
put the team ahead of individual professionals, although studies have shown that such processes can be slow and complex and also facilitated by developments in technology ${ }^{52-54}$ Tools to measure progress in team work and a safety culture could then be deployed. ${ }^{55} 56$ Of course, the role of the 'hospitalist', a doctor trained in general hospital medicine, cannot be discounted with a need to reduce the focus on specialisation. ${ }^{57}$

Second, in reflection of the above, undergraduate training and professional bodies-involved in licensing practitioners-require reorientation. Training needs to be interprofessional from the outset so students understand the interconnectivity of their roles and the nature of team work. Where implemented, often at graduate level, interprofessional training has been demonstrated to provide various benefits. ${ }^{58}{ }^{59}$ Asian and Pacific public hospitals can provide a lead on this. Debate is also needed around the role of professional colleges, often steeped in tradition and protectionist by nature. Of course, there are major challenges to this including entrenched views of various stakeholders, perceptions around loss of income for specialists with alternative models of professional organisation, and the question of how to change the professional culture.

\section{CONCLUSION}

More than ever, public hospitals in Asia-Pacific region have an opportunity to lead on local health system improvement. This article posited that the triple aim offers a solid foundation for the region's hospitals, which policymakers can apply and modify in line with their health system context and resources. The article then outlined three key areas of strategic focus-developing alliance governance, supporting innovation and reorienting the health workforce-around which public hospitals can lead debate and introduce reforms. Without such focus, there is considerable risk that the organisational and care delivery problems of the present could be exacerbated, to the detriment of patients and communities. The challenge now for the region's policymakers and leaders is to commit to this reform agenda.

\section{Acknowledgements This article resulted from a workshop on Hospital} Governance in the Asia-Pacific, supported by the Asia Pacific Observatory on Health Systems and Policies (APO), for which the authors are grateful. APO is a partnership of governments of Hong Kong SAR, The Philippines, Singapore, Thailand and The Republic of Korea as well as Asian Development Bank, World Health Organization offices for South East Asia and Western Pacific regions and the World Bank. It is hosted by WHO-SEARO. NAJ is a staff member of the WHO.

Contributors All authors contributed to discussions around the design and focus of this article. $R G$ wrote the article draft, on which the collaborating authors provided critical feedback and input. NA-J, WP and VT provided developing country perspectives.

Funding The Asia Pacific Observatory on Health Systems and Policies funded the workshop in which this article was developed.

Disclaimer The author alone is responsible for the views expressed in this publication and they do not necessarily represent the decisions or policies of the WHO.

Competing interests None declared.
Patient consent Not required.

Provenance and peer review Not commissioned; externally peer reviewed.

Data sharing statement № additional unpublished data from the study are available.

Open access This is an open access article distributed in accordance with the Creative Commons Attribution Non Commercial (CC BY-NC 4.0) license, which permits others to distribute, remix, adapt, build upon this work non-commercially, and license their derivative works on different terms, provided the original work is properly cited, appropriate credit is given, any changes made indicated, and the use is non-commercial. See: http://creativecommons.org/licenses/by-nc/4.0

\section{REFERENCES}

1. Berwick DM, Nolan TW, Whittington J. The triple aim: care, health, and cost. Health Aff 2008;27:759-69.

2. Huntington D, Hort K, eds. Public hospital governance in Asia and the Pacific. Comparative country studies, Vol. 1, No. 1. Manila: World Health Organization Asia Pacific Observatory on Health Systems and Policies, 2015.

3. United Nations Publications. Addressing the challenges of population ag eing in Asia and the Pacific: implementation of the madrid international plan of action on ageinglmplementation of the Madrid International Plan of Action on Ageing. United Nations Publications, 2018.

4. Figueras $\mathrm{J}$, McKee M, eds. Health systems, health, wealth and societal well-being. Maidenhead: Open University Press, 2012.

5. Bisognano M, Kenney C. Pursuing the triple aim: seven innovators show the way to better care, better health, and lower costs. San Francisco: Jossey-Bass, 2012.

6. Whittington JW, Nolan $\mathrm{K}$, Lewis $\mathrm{N}$, et al. Pursuing the triple aim: the first 7 years. Milbank Q 2015;93:263-300.

7. Doolan-Noble F, Lyndon M, Hau S, et al. How well does your healthcare system perform? Tracking progress toward the triple aim using system level measures. N Z Med J 2015;128:44-50.

8. Aspalter C, Yasuo U, Gauld R, eds. Health care systems in Europe and Asia. Oxon: Routledge, 2012.

9. Gauld R, ed. Comparative health policy in the Asia-Pacific. Maidenhead: Open University Press, 2005.

10. Starfield B, Shi L, Macinko J. Contribution of primary care to health systems and health. Milbank Q 2005;83:457-502.

11. World Health Organization. The world health report 2008 : primary health care - now more than ever. Geneva: World Health Organization, 2008.

12. van Weel C, Kassai R. Expanding primary care in South and East Asia. BMJ 2017;356:j634.

13. Samb B, Desai N, Nishtar S, et al. Prevention and management of chronic disease: a litmus test for health-systems strengthening in low-income and middle-income countries. Lancet 2010;376:1785-97.

14. Blouin C, Dubé L, Bertorelli E, et al. Determinants of global collective action in health: the case of the UN summit on non-communicable diseases. Global Health Governance 2014;8.

15. Weisz G, Vignola-Gagné E. The World Health Organization and the globalization of chronic noncommunicable disease. Popul Dev Rev 2015;41:507-32.

16. Nicholson C, Jackson C, Marley J. A governance model for integrated primary/secondary care for the health-reforming first world - results of a systematic review. BMC Health Serv Res 2013;13:528.

17. Satinsky M. Foundations of integrated care: facing the challenges of change. Chicago: American Hospital Publications, 1998.

18. Beech R, Henderson C, Ashby S, et al. Does integrated governance lead to integrated patient care? Findings from the innovation forum. Health Soc Care Community 2013;21:

19. Addicott R. Commissioning and contracting for integrated care. London: The King's Fund, 2014.

20. Gauld R. What should governance for integrated care look like? New Zealand's alliances provide some pointers. Med J Aust 2014;201:67-8

21. Gauld R. The theory and practice of integrative health care governance: the case of New Zealand's alliances. Journal of Integrated Care 2017;25.

22. Hearld LR, Alexander JA. Governance processes and change within organizational participants of multi-sectoral community health care alliances: the mediating role of vision, mission, strategy agreement and perceived alliance value. Am J Community Psychol 2014;53-185-97. 
23. Alexander JA, Christianson JB, Hearld LR, et al. Challenges of capacity building in multisector community health alliances. Health Educ Behav 2010;37:645-64.

24. Lovelock K, Martin G, Gauld R, et al. Better, sooner, more convenient? The reality of pursuing greater integration between primary and secondary healthcare providers in New Zealand. SAGE Open Med 2017:5:205031211770105.

25. Charles A. Developing accountable care systems: lessons from Canterbury. New Zealand: The King's Fund, 2017.

26. Timmins N, Ham C. The quest for integrated health and social care: a case study in canterbury. New Zealand. London: The King's Fund, 2013.

27. Haider D, Haider D. Cancer health alliance of metropolitan chicago. working together to achieve mutual goals. Kellogg School of Management Cases, 2017: 1-8.

28. Øvretveit J, Andreen-Sachs M, Carlsson J, et al. Implementing organisation and management innovations in Swedish healthcare: lessons from a comparison of 12 cases. J Health Organ Manag 2012;26:237-57.

29. Thakur R, Hsu SHY, Fontenot G. Innovation in healthcare: Issues and future trends. J Bus Res 2012;65:562-9.

30. Länsisalmi H, Kivimäki M, Aalto $P$, et al. Innovation in healthcare: a systematic review of recent research. Nurs Sci Q 2006;19:66-72.

31. Cresswell K, Cunningham-Burley S, Sheikh A. Creating a climate that catalyses healthcare innovation in the United Kingdom learning lessons from international innovators. J Innov Health Inform 2017;23:772-82.

32. James BC, Savitz LA. How Intermountain trimmed health care costs through robust quality improvement efforts. Health Aff 2011;30:1185-91.

33. Paulus RA, Davis K, Steele GD. Continuous innovation in health care: implications of the Geisinger experience. Health Aff 2008;27:1235-45.

34. Bohmer RMJ. Designing care: aligning the nature and management of health care. Boston: Harvard Business Press, 2009.

35. Godfrey MM, Melin CN, Muething SE, et al. Clinical microsystems, Part 3. Transformation of two hospitals using microsystem, mesosystem, and macrosystem strategies. Jt Comm J Qual Patient Saf 2008;34:591-603.

36. Kitreerawutiwong N, Jordan S, Hughes D. Facility type and primary care performance in sub-district health promotion hospitals in Northern Thailand. PLoS One 2017;12:e0174055.

37. Goodall AH. Physician-leaders and hospital performance: is there an association? Soc Sci Med 2011;73:535-9.

38. Dorgan S, Layton D, Bloom N, et al. Management in healthcare: why good practice really matters. London: McKinsey and Company/ London School of Economics, 2010.

39. Gauld R, Horsburgh S. Clinical governance assessment project: final report on a national health professional survey and site visits to 19 New Zealand DHBs. Dunedin: Centre for Health Systems, University of Otago, 2012.

40. Angood P, Birk S. The value of physician leadership. Tampa, FL: American College of Physician Executives, 2014.
41. Clark J, Nath V. Medical engagement: a Journey not an event. London: King's Fund, 2014.

42. Sligo J, Gauld R, Roberts V, et al. A literature review for large-scale health information system project planning, implementation and evaluation. Int J Med Inform 2017;97:86-97.

43. Rycroft-Malone J, Wilkinson JE, Burton CR, et al. Implementing health research through academic and clinical partnerships: a realistic evaluation of the Collaborations for Leadership in Applied Health Research and Care (CLAHRC). Implement Sci 2011;6:74.

44. Organization WH. The world health report: 2006: working together for health, 2006.

45. Irvine D. The performance of doctors: the new professionalism. Lancet 1999:353:1174-7.

46. Friedman A, Hahn KA, Etz R, et al. A typology of primary care workforce innovations in the United States since 2000. Med Care 2014;52:101-11.

47. Thomas EJ. Improving teamwork in healthcare: current approaches and the path forward. BMJ Qual Saf 2011;20:647-50.

48. Morgan S, Pullon S, McKinlay E. Observation of interprofessional collaborative practice in primary care teams: An integrative literature review. Int J Nurs Stud 2015;52:1217-30.

49. Darlow B, Coleman K, McKinlay E, et al. The positive impact of interprofessional education: a controlled trial to evaluate a programme for health professional students. BMC Med Educ 2015;15:98

50. Institute of Medicine. To err is human: building a safer health system. Washington, D.C: National Academy Press, 2000.

51. Department of Health. An organisation with a Memory. Report of an expert group on learning from adverse events in the NHS. London: Department of Health, 2000

52. de Bont A, van Exel J, Coretti S, et al. Reconfiguring health workforce: a case-based comparative study explaining the increasingly diverse professional roles in Europe. BMC Health Serv Res 2016;16:637.

53. Bridges DR, Davidson RA, Odegard PS, et al. Interprofessional collaboration: three best practice models of interprofessional education. Med Educ Online 2011;16:6035.

54. Pullon SS, Wilson C, Gallagher P, et al. Transition to practice: can rural interprofessional education make a difference? A cohort study. BMC Med Educ 2016;16:154.

55. Sexton JB, Helmreich RL, Neilands TB, et al. The Safety Attitudes Questionnaire: psychometric properties, benchmarking data, and emerging research. BMC Health Serv Res 2006;6:44.

56. Secanell M, Groene O, Arah OA, et al. Deepening our understanding of quality improvement in Europe (DUQuE): overview of a study of hospital quality management in seven countries. Int J Qual Health Care 2014;26-5-15.

57. Habicht R, Gulati M, Gulati M, et al. Hospital medicine: perspectives practices and professional development. Springer, 2017.

58. Gould K, Day KH, Barton AT. Changing student attitudes through interaction: Findings from an interprofessional workshop. $J$ Interprof Care 2017;31:540-2.

59. Joseph S, Diack L, Garton F, et al. Interprofessional education in practice. Clin Teach 2012;9:27-31. 